\title{
RADIO MOLECULAR LINE OBSERVATIONS OF LATE TYPE STARS
}

\author{
A. Winnberg \\ Max-Planck-Institut für Radioastronomie \\ Auf dem liügel 69 \\ 5300 Bonn 1 \\ Federal Republic of Germany
}

\section{Introduction}

Radio observations of spectral lines from $\mathrm{OH}, \mathrm{H}_{2} \mathrm{O}, \mathrm{SiO}$ and $\mathrm{CO}$ have put the spot-light on late-type stars and revived the interest in them. Especially they have helped the understanding of the outer envelopes of these stars and of their mass loss. They also have revealed late-type stars that are hidden from observation at optical and near-infrared wavelengths by their dense surrounding shells of gas and dust. The strong maser lines from $\mathrm{OH}, \mathrm{H}_{2} \mathrm{O}$ and $\mathrm{SiO}$ have additionally given us an excellent tool in mapping the distribution of late-type variables throughout the Galaxy. On the other hand, the interpretation of the maser amplified lines in terms of a model is difficult. Very-long-baseline interferometry (VLBI) of these lines might be a way of studying the geometry and kinematics of the envelopes but there are a few problems connected with these observations which I will point out in this paper. However, recently "thermal" microwave lines of $\mathrm{SiO}$ and $\mathrm{CO}$ have been observed from late-type stars. These lines might be easier to interpret regarding physical processes in the outer circumstellar envelopes. As so often when a new field is opening up in astronomy the solutions to several old problems have been found but at the same time many more new problems have been brought up.

In this paper $\mathrm{I}$ will review observational work on $\mathrm{OH}, \mathrm{H}_{2} \mathrm{O}$ and $\mathrm{SiO}$ maser lines and thermal emission from $\mathrm{CO}$ and SiO in Mira-type variables, late-type supergiants and carbon-stars. I will also deal shortly with some important theoretical work and model building for these objects. 
II. Mira Variables

\section{Maser Lines}

The observational status for the maser lines of $\mathrm{Oll}, \mathrm{H}_{2} \mathrm{O}$ and vibrationally excited SiO from Mira variables is summarized in an excellent way in a diagram by O1non in his Leiden Phl thesis (O1non, 1977; see Figure 1 in Olnon's contribution to this colloquium). He treated a sample of 31 maser stars - most of them Mira variables - in a statistical way. He divided these stars into two groups with colour index I - K smaller or greater than 6 . He then constructed histograms of number of stars with maser emission in a specific line at the relative velocity, $v_{\text {rel, }}$, choosing the zero point as the central oll velocity in most cases. Note that these histograms do not reflect the exact shapes of the various line profiles. They simply give the velocity distribution of the lines in the two groups of objects. We see that in the "redder" group (group 2) there are more sources emitting in the $\mathrm{OH}$ satellite line at $1612 \mathrm{MHz}$ than in the main line at $1667 \mathrm{MHz}$. (Some stars emit in the other main line at $1665 \mathrm{MHz}$ as we11.) In the "less red" group (group 1), however, there are more stars enitting at $1667 \mathrm{MHz}$. Regarding the velocity structure it is immediately apparent that group 2 has a larger separation between the two $\mathrm{OH}$ line components than group 1 . The $\mathrm{H}_{2} \mathrm{O}$ line velocities tend to fall close to the central oH velocity for group 1 and close to one or both of the OH velocities for group 2. The Sio velocity distribution seems to be unsymmetrical for group 1 (to the higher-velocity side) and to be strongly concentrated to the central velocity for group 2 .

The optical absorption line velocities usually fall close to the high Oll velocity and the emission or circumstellar line velocity close to the low Oli velocity (but this is not so well correlated as the absorption line velocities; Wallerstein, 1975). The absorption line velocity has long been regarded as the stellar velocity but recently several arguments speak against this interpretation and in favour of a stellar velocity close to the central OH velocity. The most convincing evidence for this interpretation is given by observations of thermal SiO lines in the vibrational ground state (Buhl et al., 1975; Reid and bickinson, 1976) as reported in Section II 2 . 
a) OH Lines So far only ground-state $\left({ }^{2} \Pi_{3 / 2}, J=3 / 2, v=0\right) \Lambda$ doublet lines of 011 have been observed toward Mira variables. They occur at one or several of the frequencies $1612\left(F=1^{+}-2^{-}\right), 1665$ $\left(F=1^{+}-1^{-}\right)$, and $1667 \mathrm{MHz}\left(F=2^{+}-2^{-}\right)$. No emission has ever been observed at the fourth hyperfine component frequency $1720 \mathrm{MHz}\left(\mathrm{F}=2^{+}\right.$ - $1^{-}$). A classification of these $O H$ sources which is based on the relative strengths of the $1612-\mathrm{MHz}$ line and of the main lines at 1665 and $1667 \mathrm{MHz}$ is now in wide use (Wilson and Barrett, 1972). Sources strongest in one of the main lines are called Type I Oll/IR Stars and sources strongest in the 1612-MHz satellite 1 ine are called Type II OH/IR Stars. The notation "OH/IR" originates from the time when one was not quite sure whether the $O H$ emission had something to do with the Mira phenomenon. Note that the Type I and Type II OH/IR Stars essentially conprise the same stars as in Olnon's Group 1 and Group 2, respectively (Olnon, 1977). An Oll 1 ine profile of an OH/IR star usually has a very characteristic appearance (Figure 1 and 2). It often consists of two peaks of arbitrary intensity ratio. The separation between the peaks $(\Delta V)$ is usually within the ranges $5-20$ $\mathrm{km} \mathrm{s} \mathrm{s}^{-1}$ for Type I (Figure 1) and $20-40$ $\mathrm{km} \mathrm{s}^{-1}$ for Type II OH/IR Stars (Figure 2). A very strong correlation exists between $\Delta \mathrm{V}$ and the light period of these stars: a larger $\Delta \mathrm{V}$ is associated with a longer period (Dickinson et al., 1975). Harvey et

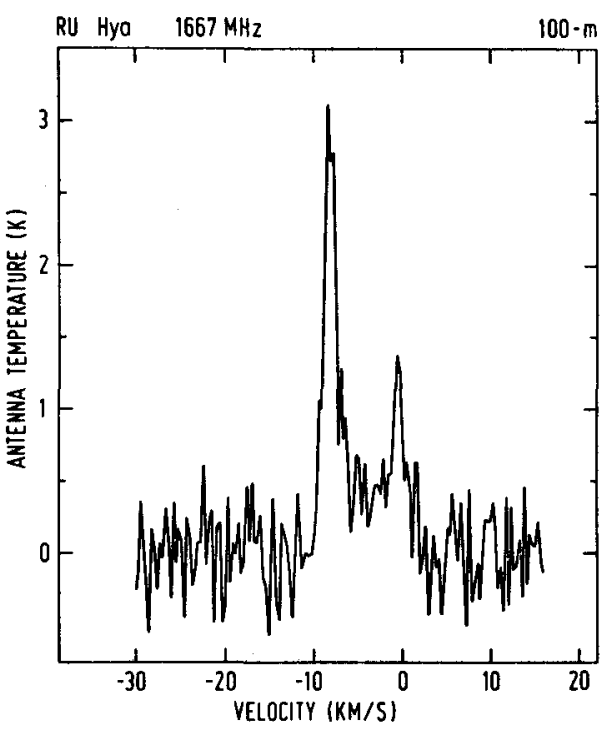
a1. (1974 a) showed that the $\mathrm{OH}$ flux varies in phase with the

Fig. 1 A 1667-MHz OH line profile of a Type I OH/IR Star (RU Hya) observed with the 100-m telescope 
infrared flux but with a phase lag of $\sim 0.1-0.2$ period compared with the optical flux strongly suggesting that the $\mathrm{OH}$ molecules are pumped by IR radiation.

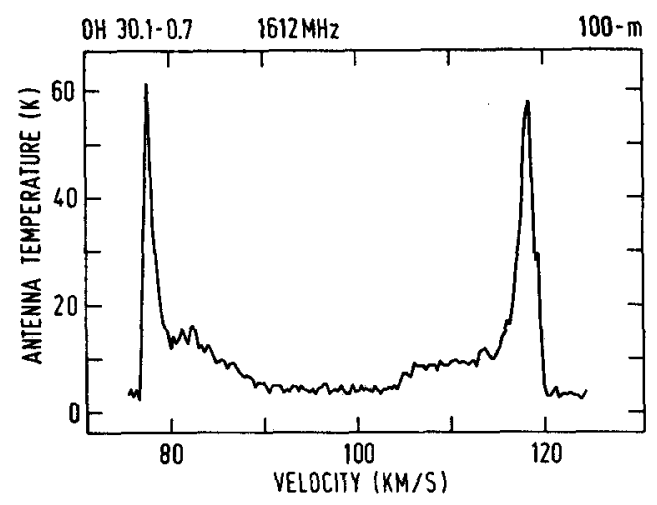

Fig. 2 A 1612-MHz OH line profile of a Type II OH/ IR Star $(0 \mathrm{H} 30.1-0.7)$ observed with the 100m telescope

For two earlier review papers on OH/IR work see Caswell (1974) and Hyl and (1974).

A further recent result on the OH-light-curve relation has been given by Bowers (1975) and Bowers and Kerr (1977). They find from a comparison of the light curves of Mira variables with and without $O H$ emission that the OH Miras have smaller rise-time-to-period ratios than non-OH Miras, i. e. OH Miras have more asymmetric light curves than non- $\mathrm{OH}$ Miras. They also show that for Miras in general stars with longer periods have a slightly smaller rise-time-to-period ratio than stars with shorter periods. They suggest that shock waves may play an important role in the mass loss mechanisin of Miras (see e. g. Slutz, 1976) with long periods and oll emission and they estimate that the OH Miras contribute $z 20$ per cent of all mass lost by stars.

The most powerful observations of maser line sources in general are VLBI observations. The problems of such observations applied to the 
OH Wira sources are twofold: 1. They are comparatively insensitive since one cannot integrate for a long tilie. 2. Most source diameters are too large ( $>0.3^{\prime \prime}$ ) for available baseline lengths. Ideal baselines would be $<100 \mathrm{~km}$ and $\mathrm{strange}$ enough there are not many radio telescopes so close to each other. The first interferometer observations therefore concentrated on late-type supergiants with oll emission since this emission is generally stronger than the one from the Miras (see Section III). The first positive results on Ol Mira stars were obtained by Reid et a1. (1977). They studied three stars at $1612 \mathrm{Mltz}$ in great detail: U Ori, R AqI and IRC+10011 (or WX PSC). For these stars they found that the maser components showed a complex structure which they could interpret in terms of "core-halo" models. However, their data are also consistent with a "multiple component" model. Figure 3 taken from their paper illustrates these two interpretations schematically. In the top drawing the "cores" of the low- and high-velocity components are denoted by the small solid and open circles and the "halos" are denoted by the hatched areas. Since the "halos" represent the flux they could not detect (in most cases the larger part of the flux) because of too high spatial resolution, it is not certain that they are centred exactly around the "cores". In the bottom drawing the multi-component model is illustrated. Maser components in the low- and highvelocity enission complexes are represented by closed and open circles, respectively.

For IRC+10011, for example, they found that the "cores" are smaller than 0.04 " and that the "halos", or alternatively the cluster size of the "cores" in the multicomponent model, are larger

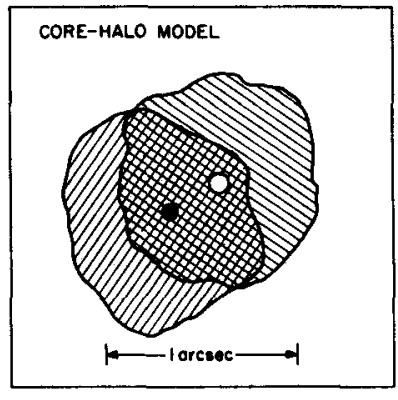

MULTIPLE COMPONENT MODEL

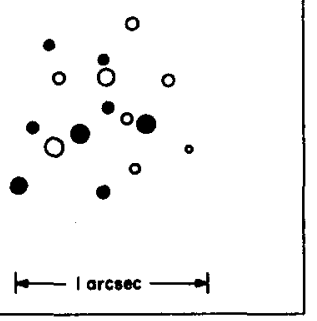

Fig. 3 Schematic representation of the "core-halo" and "multi-component models of Reid et al. (1977). From Reid et al. (1977) 
than $0.4^{\prime \prime}$. The angular separation between the ligh- and low-velocity "cores" in the "core-halo" model is less than $\sim 0.2 "$ ". Only $\sim 1$ per cent of the total flux is coming from the "cores".

Personal1y, I think that the $\mathrm{OH}$ structure of these stars ultimately will turn out to be so complex that a combination of the two models will be necessary in order to describe it, i. e. several "cores" and "halos" will have to be superimposed on top of each other.

Such a picture had to be adopted for the unusual star U Ori by Reid et al. (1977). The Oll emission was discoverd by wilson et al. (1972) in 1969. It then showed main-line emission and no 1612-MHz emission stronger than $0.5 \mathrm{Jy}\left(1 \mathrm{Jy}=10^{-26} \mathrm{~W} \mathrm{~m}^{-2} \mathrm{~Hz}^{-1}\right)$. Sometime between Nov. 1973 and May 1974, however, the star brightened at $1612 \mathrm{MHz}$ (Pataki and Kolena, 1974) and disappeared at the $1667 \mathrm{MHz}$. The 1612MHz line spectrum now consists of at least 6 line components of which at least one is strongly polarized. Such properties are unique for an OH Mira and it is the only case in which such a dramatic change has been reported for a stellar OH maser: the star changed its class from Type I to Type II OH/IR. Therefore we must bear in mind that the VLBI map of the 1612-MHz ellission from U Ori may not be typical for an ordinary $\mathrm{OH}$ Mira star. Figure 4 shows the relative positions (the phase stability was not good enough to allow a determination of the absolute position of the source cluster) of 12 "cores" each smaller than $~ 0.03 "$. The numbers give the radial velocities of the various spectral components and the crosses show the positional errors. The feature at $-44.8 \mathrm{~km} \mathrm{~s}^{-1}$ served as a reference. The map does not show maser components larger than $\sim 0.3^{\prime \prime}$ and some fraction $(\sim 1 / 3)$ of the total flux seens to come from such components. The linear scale in Figure 4 is based on a trigonometric distance of $190 \mathrm{pc}$ (Wilson and Merri11, 1942).

I want to add to this picture of U Ori that the angular diameter of the star has been measured by lunar occultation at $\lambda=2 \mu$ (Ridgeway et al., 1977). The diameter for a uniformly enitting disk is $15.5 \pm 0.3$ milliarc seconds. For a distance of $190 \mathrm{pc}$ it means that the star has a linear size of $\sim 4 \times 10^{13} \mathrm{~cm}$ whereas the $\mathrm{OH}$ enitting envelope is larger than $\sim 9 \times 10^{14} \mathrm{~cm}$. So we see that the oll envelope is larger than the central star by a factor of at least 10 . However, we do not know with sufficient accuracy the relative position of the star and the OH sources. This would be known only if we could determine 
the absolute position of the on sources to better than $\sim 0.1 "$. Such measurements and measurements of the "halo" diameters are currently under way with the Jodrell Bank-Chilbolton interferometer system (Booth, private communication).

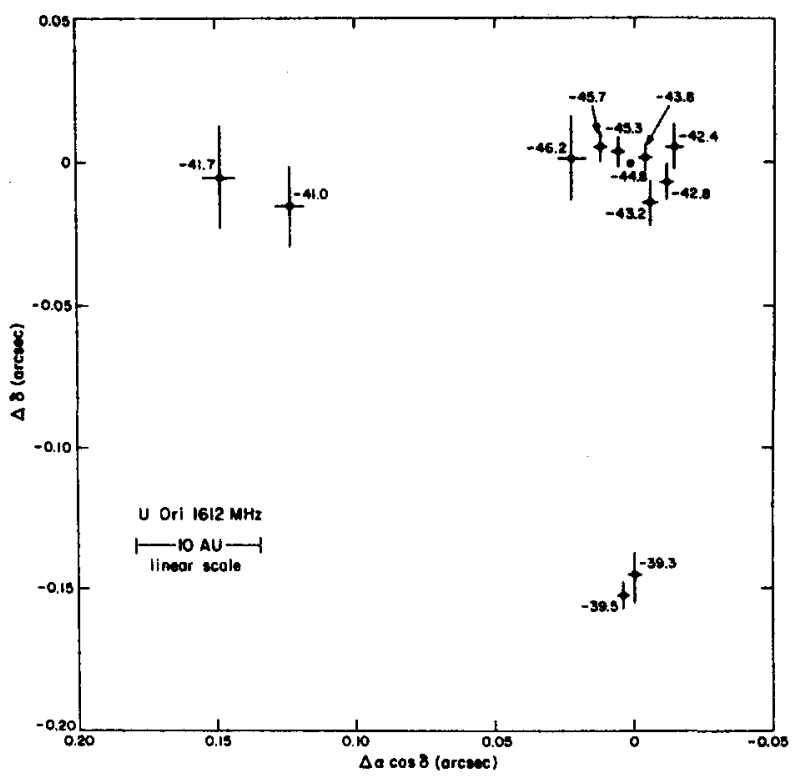

Fig. 4 Map of the 1612-MHz enission of $U$ Ori. The crosses denote the relative positions of the "cores" with their $2-\sigma$ errors. The numbers at the crosses denote the radial velocities in $\mathrm{km} \mathrm{s}^{-1}$. The reference component is at $-44.8 \mathrm{~km} \mathrm{~s}^{-1}$. The linear scale is based on a trigonometric distance of 190 pc. From Reid et a1. (1977)

As for possible tiodels of the OH emission from Miras one can very easily rule out contracting or rotating circumstellar envelopes. The mass of the central star for such models has to be of the order of $100 \mathrm{M}_{\odot}$ or more (see Reid et al., 1977). The only possible model is that of an expanding envelope. This is the most plausible model also because we all know that there is a considerable mass loss from these stars ( $10^{-6} \mathrm{M}_{\odot} \mathrm{yr}^{-1}$ ) probably driven by radiation pressure on dust 
grains. Since the stellar velocity probably is midway between the on velocities a symmetric expansion model where the low- and high-velocity OH enission is originating on the front- and back-side of the envelope is to be preferred as compared to expansion models involving shock fronts (Wilson et al., 1970). Figure 5 again taken from Reid et al. (1977) illustrates this model conception. Since the paths of maximum maser gain are those in which the radial velocity gradient has a mininum, then for constant expansion velocity the emission in this model should be confined to a small "cap" directly in front and back of the star. Maser emission from the limb is not expected in general because the velocity gradient is greatest there. One would expect the low- and high-velocity OH emission components to appear spatially coincident. The VLBI-observations of IRC+10011 (Reid et a1., 1977) are probably consistent with this prediction.

The simple expanding shell nodel predicts that maser components, of a given velocity relative to the central star, $v$, should be located on a ring concentric with the star and with a radius

$$
a(v)=R \sin \theta=R\left[1-\left(v / v_{e}\right)^{2}\right]^{1 / 2}
$$

where $R$ is the mean radius of the oH-emitting envelope, $v_{e}$ is the expansion velocity and $\theta$ is defined in Figure 5. It is impossible to test this model on the basis of available data, since information on the lower spatial frequencies is absent to a large degree. One possible method which could be used as a complement to interferometric methods is to use lunar occultations. At $\lambda=18 \mathrm{~cm}$, the angle between the geometrical lunar shadow and the first Fresnel maximum would be $\sim 4 "$. This means that one would expect to observe Fresnel diffraction patterns for sources smaller than this limit. Even for sources larger than 4", however, one would be able to get a measure of the angular size by measuring the slope of the occultation curve. The aperture of the telescope would put a lower limit on the neasurable size, but even for the 100-m telescope this limit would be $\sim 0.05 "$. of course lunar occultation techniques would work equally well for $\mathrm{H}_{2} \mathrm{O}$ and $\mathrm{SiO}$ stellar masers. However, I am not aware of any observations of this kind to date.

It is very likely that the picture of a Mira in the OH lines will not turn out so simple for several reasons:

1. The shell may fragment into many small clouds as it expands owing to inhomogeneities present during the formation and initial acceleration. 
a)
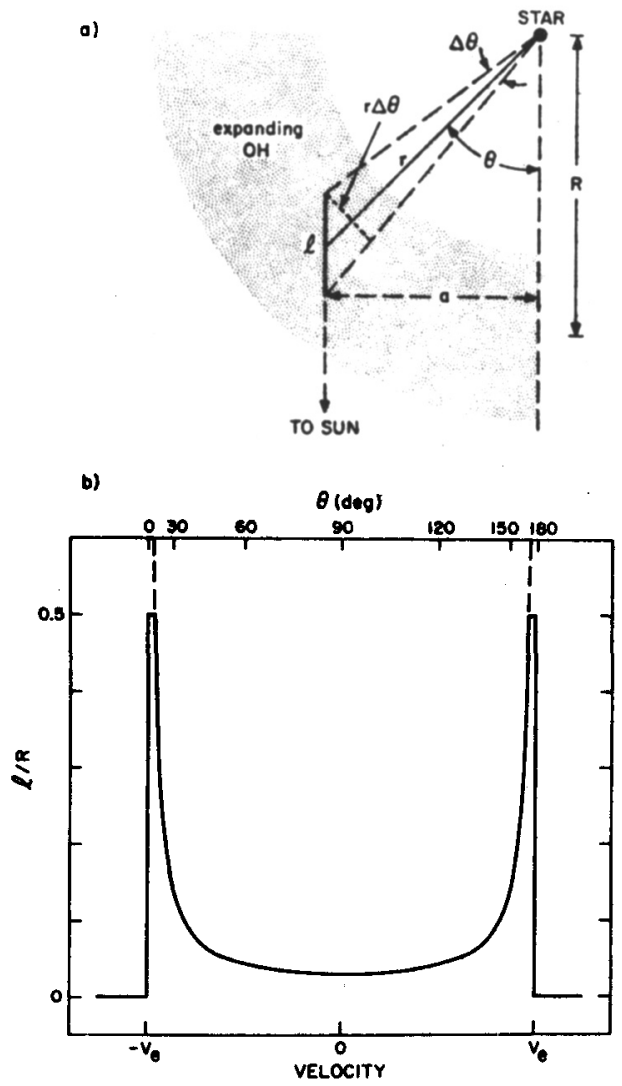

Fig. 5 a) A schematic diagram of the expanding circumstellar shell model for stellar $\mathrm{OH}$ maser emission. The path length of maser amplification, 1 , along a ray through a shel1 of mean radius, $R$, is defined in terms of radial distance to the star, $r$, and position in the she11, $\Theta$ or a. b) The path length of maser amplification, 1 , normalized to the mean shell radius, $R$, versus the observed radial velocity. A constant circumstellar expansion velocity, v, is assumed. 1 is determined by equating the radial-velocity differential along the ray with the thermal Doppler width of the maser line. The plot $1 / R$ is cut of where 1 equals $0.5 \mathrm{R}$ which is the assumed thickness of the shell. From Reid et al. (1977) 
2. Shells may be ejected more or less irregularly because the condensation and acceleration of dust grains near the central star are probably critically tied to the temperature cycle of these stars.

3. It is possible that the oH emission lines are originating in several circuristellar shells with slightly different expansion velocities. There would not then be a simple relation between the radial velocity and the angular distance from the star.

Another observing proposal for OH Mira stars has been suggested by Dr. Sherwood (private comunication). By monitoring these sources with high sensitivity one should be able to detect (or not detect!) a phase difference between the low- and high-velocity feature. If the diameter of an OH Mira envelope is of the order of $10^{17} \mathrm{~cm}$, then one would expect a phase lag of about 1 month of the high-velocity feature as compared to the low-velocity feature. This would be difficult to detect in periods of typically 400 - 700 days but is nevertheless worth an at tempt.

A number of important theoretical articles have been written recently. Unfortunately, I will not have time to discuss them in detail. The papers by Goldreich and Scoville (1976) and by Elitzur, Goldreich and Scoville (1976) build a very sophisticated model of a Mira surrounding. The first paper deals with the chemistry, dynamics and thermodynamics of an expanding shell. In the second paper, a model for the circumstellar maser mechanism is developed. The 1612-MHz maser is powered by the absorption to the $2_{1 / 2}, J=5 / 2$ rotationally excited state. The subsequent radiative cascade inverts the $1612-\mathrm{MHz}$ levels provided that the last rotational transition (from ${ }^{2} \Pi_{1 / 2}, J=1 / 2$ to ground) is optically thick. Inversion of the main lines can be achieved if the temperature is high enough ( $2500 \mathrm{~K}$ ) or there is enough turbulence to allow partial overlap of infrared rotational lines to take place (Litvak and Dickinson, 1972).

The work by Kwok (1976) gives a simple model of OH and $\mathrm{H}_{2} \mathrm{O}$ masers around late-type stars. He also discusses the scattering of optical photons by the expanding dust and shows that this mechanism would cause a red-shift (or better "red-skew") of the optical photospheric lines. Unfortunately, this theory is not yet worked out in full detail. 
Olnon (1977) in his thesis work takes optical depth effects into account and shows that it has a considerable effect on the velocity field in the envelope. See paper given at this colloquium.

b) $\underline{H}_{2}$ olines Emission at $\lambda=1.35 \mathrm{~cm}$ from water vapour is observed towards several lilira variables preferably from Type $I$ and from Type II sources with strong $\mathrm{OH}$ main-1ine emission. Thus the occurence of $\mathrm{H}_{2} \mathrm{O}$ emission seems to be correlated with the presence of ol main-line emission. However, there are some stars with $\mathrm{H}_{2} \mathrm{O}$ emission but without detected OH emission. Most stellar $\mathrm{H}_{2} \mathrm{O}$-emission lines have a sinple profile (Figure 6 a). However, multiple-component line profiles are not uncommon (Figure $6 \mathrm{~b}$ and $c$ ) and an especially interesting class

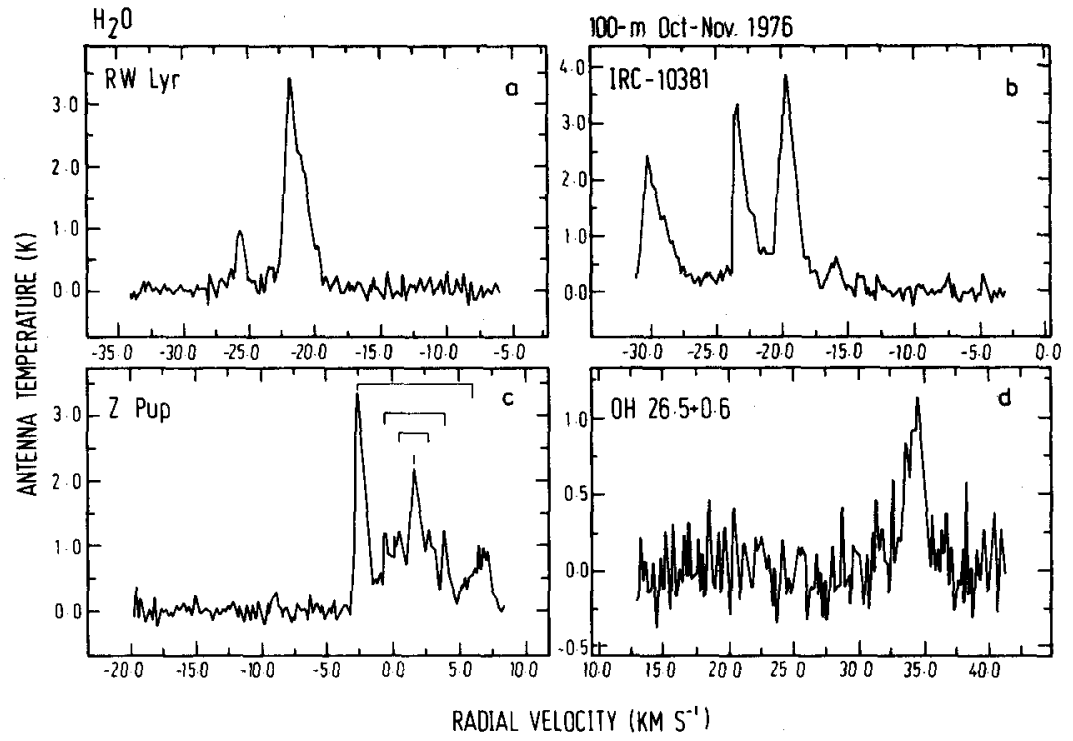

Fig. 6 Examples of $\lambda=1.35 \mathrm{~cm} \mathrm{H}{ }_{2} \mathrm{O}$ line profiles from 3 Mira variables and the peculiar $Z_{H} / I R$ source OII $26.5+0.6$. The observations are made with the 100-m telescope (Baud, Habing, olnon, Schultz, and Winnberg, to be published).

of $\mathrm{H}_{2} \mathrm{O}$-spectra are those with a certain degree of symmetry around a central line component (Figure $6 \mathrm{c}$; Knowles and Batchelor, 1976). 
Such $\mathrm{H}_{2} \mathrm{O}$ spectra are often found in maser sources associated with very young objects (compact HII regions, compact IR sources; Genzel and Downes, $1977 \mathrm{a}$ ). They may be interpreted as originating from sources with several expanding shells. $\mathrm{H}_{2} \mathrm{O}$ emission is seldom found in $011 / \mathrm{IR}$ sources detected in $1612-\mathrm{MHz}$ OH surveys. For example, the $\mathrm{H}_{2} \mathrm{O}$ line in the very strong Type II OH/IR Star OH26.5+0.6 (Andersson et al., 1974) is quite weak (Figure 6 d). The $\mathrm{H}_{2} \mathrm{O}$ line detected is close to the high-velocity of line $\sim+40 \mathrm{~km} \mathrm{~s}^{-f}$ but on the low-velocity side.

Dickinson and Kleinmann (1977) have observed double-1ine structure in $\mathrm{H}_{2} \mathrm{O}$ lines from long-period variables plus NML Cyg which is probably a supergiant (see Section III). The $\mathrm{H}_{2} \mathrm{O}$-line components always lie close to but on the inside of the OH-Iine components (Figure 7). This similarity strongly suggests that the geometry of the enitting regions is she11-like and that the stellar velocity is near the midpoint of the two emission peaks. In asymmetric models where the oll lines are produced at two discrete regions on the near side of the star one would not a priori expect symmetry of the $\mathrm{H}_{2} \mathrm{O}$-line velocities with respect to the OH-line velocities. Since it is likely that the circumstellar gas velocity increases monotonically with the distance from the star, then the $\mathrm{H}_{2} \mathrm{O}$ emission originates in a shell closer to the star than the 1612-MHz OH enission. One may infer this also from the high excitation $\left(447 \mathrm{~cm}^{-1}, \sim 600 \mathrm{~K}\right)$ of the energy levels $\left(6_{16}-5_{23}\right)$ involved in the $1.35-\mathrm{cm} \mathrm{H}_{2} \mathrm{O}$ transition. Stellar water masers must therefore originate in relatively hot regions. This picture is also consistent when one considers maser emission from main-line $\mathrm{OH}$ and from vibrationally excited SiO (see next section). In order to produce main-line $\mathrm{OH}$ enission one needs an environment which is hotter than required for 1612-MHz radiation (see previous section; Litvak and Dickinson, 1972). Main-line enission velocities, in fact, always fall within the $1612-\mathrm{MHz}$ line velocities. The first vibrational state of SiO has an excitation energy in excess of $1200 \mathrm{~cm}^{-1}(Z 1700 \mathrm{~K})$. Sio-maser velocities fall at or within the $\mathrm{H}_{2} \mathrm{O}$ velocities, suggesting that the SiO masers are situated still closer to the photosphere of the star itself.

The $\mathrm{H}_{2} \mathrm{O}$ flux varies in phase with the IR flux just as the $\mathrm{OH}$ flux does (Schwartz et al., 1974). However, the flux of individual $\mathrm{H}_{2} \mathrm{O}-$ line components may vary relative to each other in a more irregular way during the period of the star. Infrared pumping through the 2.7 and $6.2 \mu$ vibration-rotation bands (Litvak, 1969) seems to be the 


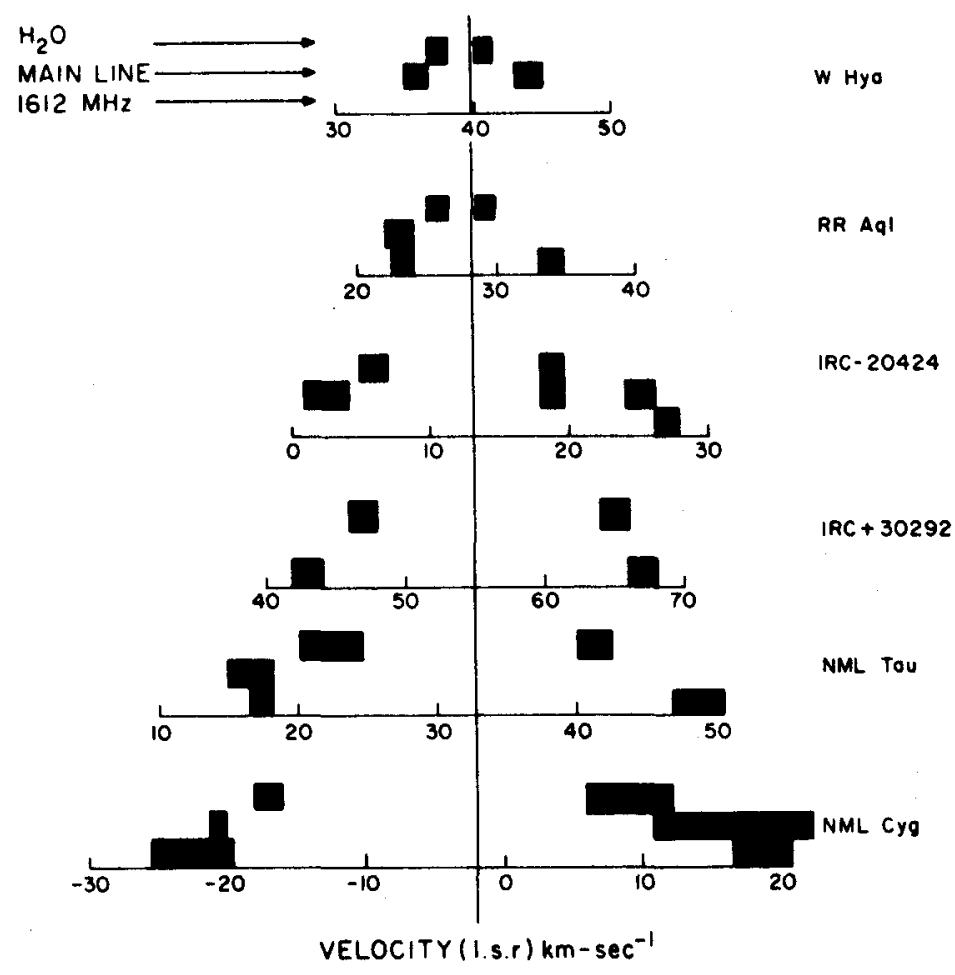

Fig. 7 Maser radial velocities for $\mathrm{H}_{2} \mathrm{O}$, OH nain 1 ines and OH $1612-\mathrm{MHz}$ emission for the indicated stars. From Dickinson and Kleinmann (1977)

most probable mechanism.

c) Sio lines Calculation of chemical equilibriun in cool stars (e. g. Goon and Auman, 1970) indicate that SiO should be an abundant molecule in the atmospheres of late-type giants and supergiants. In fact the vibration-rotation bands of sio at $\lambda=4 \mu(\Delta v=2)$ have been observed in several late-type stars (see Hinkle et al., 1976). Still the discovery of microwave lines from Sio was a pure accident (Snyder and Buh1, 1974; Kaifu et a1., 1975; Snyder and Buh1, 1975)! Recent surveys for SiO microwave lines from late-type stars have been 
made by Blair and Dickinson (1977) and by Balister et al, (1977) so that we now know $\sim 40$ Mira variables and late-type supergiant stars with SiO-microwave emission.

The transitions involved in the radio lines are all between rotational levels within the same vibrational state. Lines observed from vibrationally excited states are all due to maser amplification. In the $v=1$ vibrational state, the $J=1-0(43.1 \mathrm{GHz}$; Snyder and Buh 1 , 1975), 2-1(86.2 GHz; Kaifu et al., 1975), and $3-2(129.4 \mathrm{GHz}$; Davis et al., 1974) transitions have been observed and in the $v=2$ state the $J=1-0(42.8 \mathrm{GHz}$; Buhl et a1., 1974) transition. In the vibrational ground state $(v=0)$ no maser amplified lines have been observed in Miras (see next section). Kwan and Scoville (1974) have presented a pumping model based on vibrational transitions from $v=0$ to $v=1$ and $v=2$ at 8 and $4 \mu$, respectively. Geballe and Townes (1974) mention a number of close coincidences of vibrational transitions in Sio which alter the radiation field and provide the necessary population inversion. One of their suggestions involves the pumping of the Sio molecule by the overlap of the $1219.1 \mathrm{~cm}^{-1}$ line of $\mathrm{H}_{2} \mathrm{O}$ with the $2-1 R(0)$ transition of SiO.

In their survey for Sio emission, Blair and Dickinson (1977) find Sio in the type S star, $W$ And, and in $T$ Cep which is a borderline case between type $M$ and type $S$. Sio was already known to originate from $x \mathrm{Cyg}$, another S-type star. Neither $\mathrm{OH}$ nor $\mathrm{H}_{2} \mathrm{O}$ emission has been observed from any $S$ star so far. The authors think that this has to do with the higher $\mathrm{C} / \mathrm{O}$ ratio in the $\mathrm{S}$ stars as compared to the $M$ stars. They also show that for all Sio stars, with the exception of the source in Orion, there are always more photons at $\lambda=4.8 \mu$ than at $43.1 \mathrm{GHz}$ (the frequency for the transition $v=1, J=1-0$ ). This strongly supports the hypothesis of 4 and $8-\mu$ pumping. The SiO source in Orion is probably not associated with any late-type star. There are several reasons for believing this. The Sio radial velocity, for example, is nicely correlated with the velocities of $\mathrm{OH}$ and $\mathrm{H}_{2} \mathrm{O}$ sources associated with the KL nebula (Genzel and Downes, $1977 \mathrm{~b}$ ) and the $\mathrm{SiO}, \mathrm{OH}$, and $\mathrm{H}_{2} \mathrm{O}$ positions all coincide to several arc seconds (Moran et al., 1977a; Hansen et $21 ., 1977)$. Since the $\mathrm{OH}$ and $\mathrm{H}_{2} \mathrm{O}$ spectra have properties typical for sources associated with HII regions it is unlikely that the Sio source is related to a late-type star.

Balister et al. (1977) find that the intensity variation of Sio sour- 
ces is complicated. They observed strong day-to-day variations in some cases. Also the entire line profile seems to undergo significant changes (Figure 8). The line profiles of different transitions of the
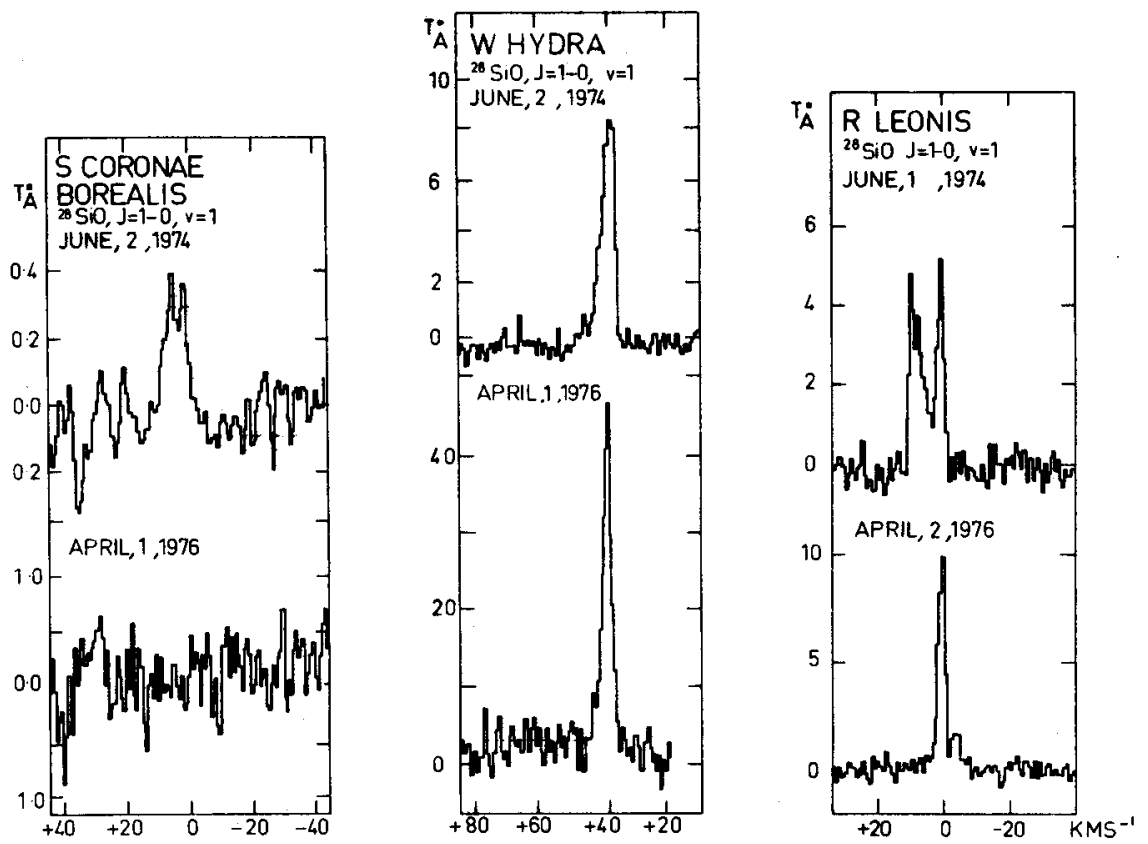

Fig. 8 Sio maser emission lines of the $v=1$, $J=1-0$ transition in three Miras. The top spectra (from 1974) are from Snyder and Buhl (1975) and the bottom spectra (from 1976) are taken by Winnewisser and Churchwell (to be published)

same star observed at the same time have often no relation to each other. The peak flux density of various Sio lines of the same source usually are similar and sometimes the higher excited lines are stronger than the lower excited lines.

\section{Therma1 Lines}

Recently the increased sensitivity of radio receivers at millimeter 
wavelengths has allowed the detection of non-maser emission from sio in several oxygen-rich objects (Buhl et al., 1975) and from $C 0$ in a number of carbon- and oxygen-rich objects (Zuckerman et a1., 1977). There is every reason to believe that such measurements can be extended to a large number of objects. Non-maser emission lines may provide the most powerful means for determining such important physical parameters as the mass loss rate and the fractional abundances of many common molecules in circumstellar envelopes.

Unlike the vibrationally excited Sio lines which are maser amplified, the ground state $(v=0)$ lines seem to be thermal and very broad $\left(220 \mathrm{~km} \mathrm{~s}^{-1}\right)$. Reid and Dickinson (1976) determined the stellar radial velocities by analysing the ground-state Sio lines ( $v=0$, $J=2$ - 1) observed by Buh1 et a1. (1975). In a spherically expanding model the central line velocity must be equal to the stellar velocity and half the total line width must be equal to the expansion velocity. Reid and Dickinson (1976) found that the stellar velocity always falls close to the central OH velocity.

Morris and Alcock (1977) have investigated the excitation of the ground-state Sio lines in the expanding envelope of cool stars. They also present a model of line intensities and profiles. One would get 5 important pieces of information from observations of the non-maser Si0:

1. The line centre velocity, which immediately yields the stellar velocity.

2. The full line width which leads immediately to the asymptotic expansion velocity.

3. The shape of the $J=1$ - 0 1ine, which gives an indication of the mean optical depth of this line.

4. The absolute intensity of a given line.

5. The relative line intensities of different rotational transitions. Measurements of 3,4 , and 5 can in principle provide a determination of $f \dot{M}, W$ and $D$, where $f$ is the mass fraction of SiO, $\dot{M}$ the mass loss rate, $W$ the dilution factor of $8-\mu$ flux, and $D$ the distance to the star.

Zuckerman et a1. (1977) have detected the $J=1-0$ line of $C 0$ in seven (and probably eight) carbon stars (see section IV) and in two (and probably three) oxygen-rich stars. Although emission from interstellar co sometimes contaminates the observations the authors claim 
that it is possible to distinguish the circumstellar radiation because of its much larger linewidth ( $20 \mathrm{~km} \mathrm{~s}^{-1}$ as compared to $\lesssim 5 \mathrm{~km} \mathrm{~s}^{-1}$ ). Essentially the same possibilities of physical interpretation seem to exist for the co lines as for the thermal sio lines.

Recently McGee, Balister and Newton (1977 a) have detected the $J=4+31$ ine of $\mathrm{HC}_{3} \mathrm{~N}$ in $\mathrm{W}$ Hya (a Mira) and McGee, Newton and Brooks $(1977 \mathrm{~b})$ have detected the $\mathrm{J}=1 \rightarrow 0$ line of $\mathrm{HC}_{5} \mathrm{~N}$ in the same star. Both lines seem to be thermal and their radial velocities are consistent with the thermal Sio velocities (Reid and Dickinson, 1976). It is to be expected that these lines will be observed in more stars and that other organic molecules will be detected in Mira envelopes.

\section{Supergiants}

Whereas the Mira variables seem to constitute a fairly homogeneous group of objects, the supergiants of late spectral type are true individuals. To date six or seven of these objects with microwave emission are known. All of them show maser emission in the $\mathrm{OH}, \mathrm{H}_{2} \mathrm{O}$ and SiO lines. One of them (NML Cyg) even has a weak Oll line from an excited state $\left({ }^{2} \Pi_{3 / 2}, J=5 / 2\right)$. The ground-state OH lines usually are strongest at $1612 \mathrm{MHz}$, but there are two exceptions (S Per and AH Sco) which are strongest in the main lines (Baudry et al., 1977). The main lines are usually circularly polarized, a fact which suggests the presence of magnetic fields close to the stars. The $O H$ emission usually show two main components, as for the Miras, but the separation is usually $z 30 \mathrm{~km} \mathrm{~s}^{-1}$. The $\mathrm{H}_{2} \mathrm{O}$ line profiles are mostly very complex and show the kind of symmetric structure I mentioned earlier. The Sio spectra show a similar tendency but do not cover such a large velocity range as the $\mathrm{H}_{2} \mathrm{O}$ lines.

It is not quite clear whether late-type supergiants with microwave maser emission are pre-main-sequence objects or evolved stars. Herbig (1969) suggested that the strong $\mathrm{OH}, \mathrm{H}_{2} \mathrm{O}$ and $\mathrm{SiO}$ source $\mathrm{VY} \mathrm{CMa}$ is a proto-planetary system consisting of a central star and an expanding disk-shaped nebula. In fact he was the first to propose that the $\mathrm{OH}$ emission is coming from the front and back side of an expanding shell and that the optical spectrum of the star is red-shifted and broadened due to scattering by the escaping dust particles.

The 1612-MHz OH line spectrum of VY CMa is truly "enormous" (Fig. 9). 


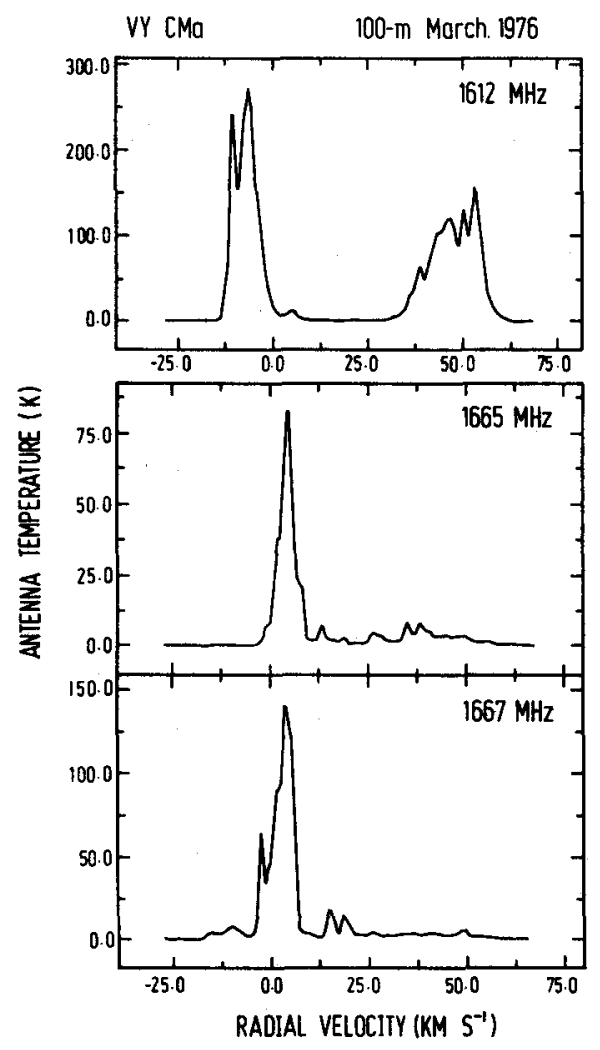

Fig. 9 The 1612,1665 , and $1667-\mathrm{MHz}$ OH 1ine profiles of VY CMa observed with the 100-m telescope (Booth and Winnberg, to be published)

It consists of two very broad line complexes separated by $\sim 60 \mathrm{~km} \mathrm{~s}^{-1}$. However, there are numerous secondary peaks present. The main-line spectra are also complicated and have velocities intermediate to the $1612-\mathrm{MHz}$ velocities. These 1 ines are circularly polarized. The $\mathrm{H}_{2} \mathrm{O}$ line spectrum shows an interesting symmetrical structure with a central component at $\sim 20 \mathrm{~km} \mathrm{~s}^{-1}$ and several components placed symmetrically above and below the central component (Figure 10). The appearance of this line profile, however, can vary drastically with 


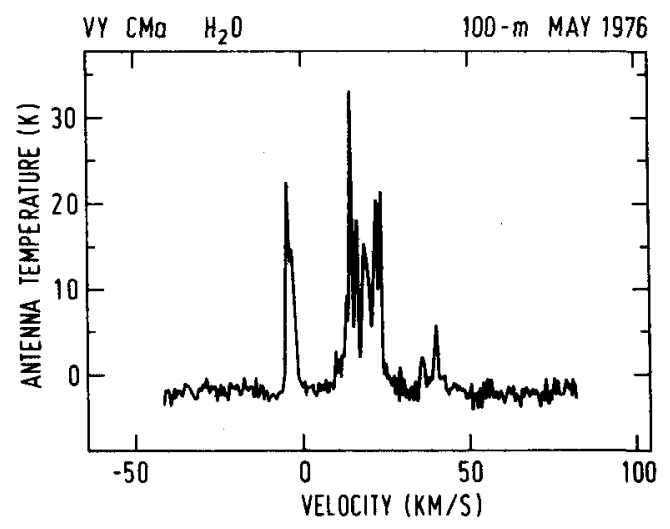

Fig. 10 The $\mathrm{H}_{2} \mathrm{O}$ line profile of VY CMa observed with the 100-m telescope (Downes and Genzel, to be published)

time. The Sio-line profiles, finally, also show a kind of symmetrical structure (Figure 11). This triple structure has been interpreted by Van Blerkom and Auer (1976) as originating in a rotating equatorial disk seen nearly edge-on. Perhaps the $\mathrm{H}_{2} \mathrm{O}$ spectrum could be interpreted in the same (although somewhat more complicated) way.

Interferometer maps of VY CMa have been produced by Masheder et al. (1974), Reid (1975), and by Moran et al. (1977 b). Due to differences in $u-v$ coverage, spectral resolution etc. the three maps differ in details somewhat but the gross picture is the same (the coordinate axes in the map by Masheder et al. (1974), however, should be inverted!). Moran et al. $(1977$ b) fitted their data to a simple expanding shell model. In this model the oll spots are assumed to originate from points on a thin spherical shell which is expanding radially away from the star. The model has five parameters: $\theta_{x 0}$ and $\theta_{\text {yo }}$, the coordinates of the centre of expansion, which presumably are the stellar coordinates; $v_{0}$, the radial velocity of the star; and $R$, the radius of the she11. The velocity of the i'th maser spot is: 


$$
v_{i}=v_{0}-v_{E} \cos \psi_{i}
$$

where $\Psi_{i}$ is the polar angle between the line of sight to the observer and the $i$ 'th maser spot as seen from the star. The projected distance from the star to the $i$ 'th maser spot is then:

$$
d_{i}=R\left[1-\left(\frac{v_{i}-v_{0}}{v_{E}}\right)^{2}\right]^{1 / 2}
$$

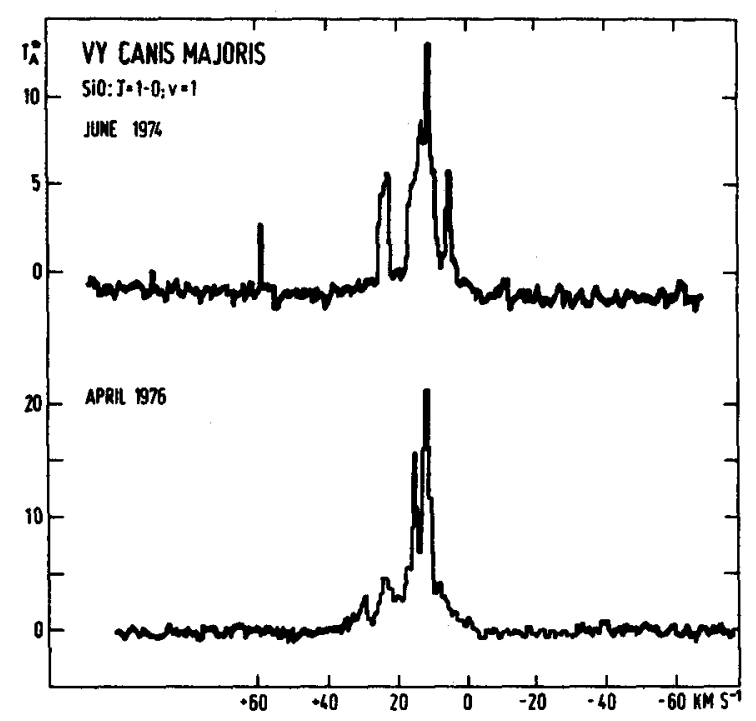

Fig. 11 The $v=1, J=1-0$ Sio line profile of $\mathrm{VY} \mathrm{CMa}$ from two different epochs. The upper spectrum is from Snyder and Buh1 (1975) and the lower spectrum from Winnewisser and Churchwell(to be published)

Solutions were sought which minimized the mean-square error between the projected distances of the spots in the model and the data. For the 13 strongest features, they found a solution as depicted in Figure 12. This places the star some $4^{\prime \prime}$ to the east of the oll maser spots and says that the velocity of the star should be $21 \mathrm{~km} \mathrm{~s}^{-1}$ which is midway between that of the low-velocity and that of the high-velocity $\mathrm{OH}$ components. Incidentally this is close to the velocity measured by Wallerstein (1977) from optical lines. The expansion velocity is 


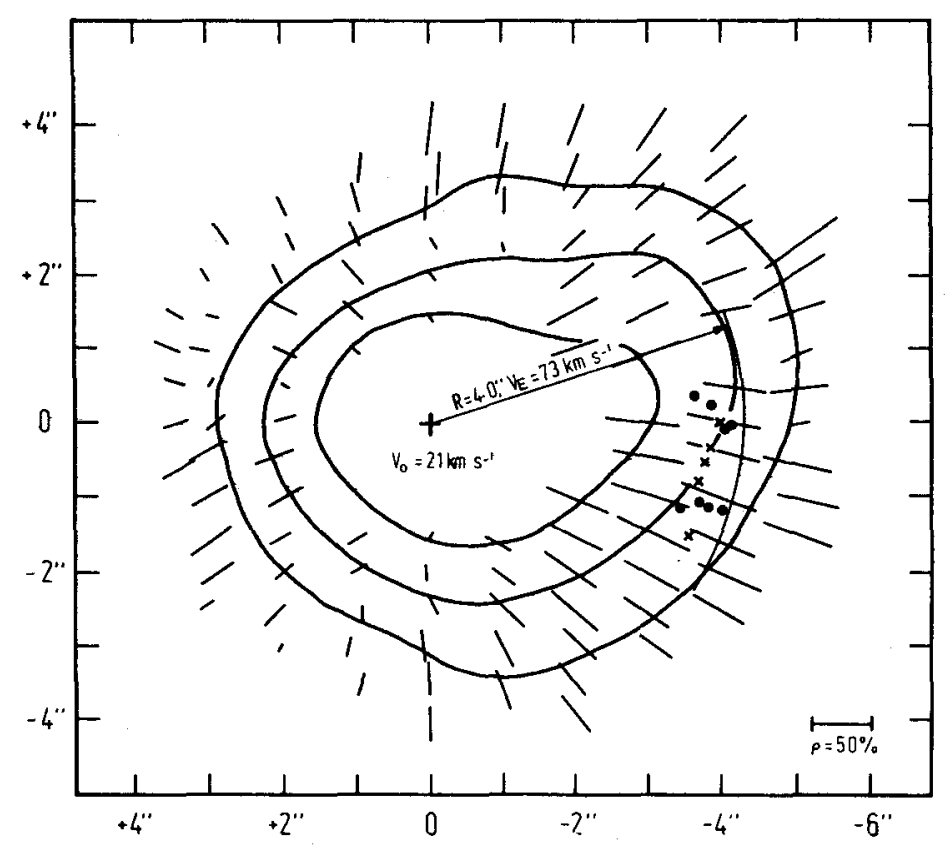

Fig. 12 Map of the positions of the 13 principal 1612-MIIz OH features in VY CMa and the best-fitting thin shell model after Moran et al. $(1977$ b) superimposed on the map of the reflection nebula surrounding VY CMa as mapped by lierbig (1972). The contours connect points of equal unpolarized flux at 17,50 , and 150 arbitrary units above the background. The length of the straight lines is proportional to the degree of linear polarization according to the scale in the lower right corner and the direction of the lines is perpendicular to the E-vector. The centre of expansion in the model of Moran et al. (1977 b) and the position of the star have been brought to coincidence.

$73 \mathrm{~km} \mathrm{~s}^{-1}$ which is quite high but perhaps not unrealistic considering the high luminosity of the star. It is somewhat unexpected that all the maser spots are close to the tangential point and occupy a very small part of the shell. We must remember that this model fit is very uncertain and inight be totally wrong. llowever, it is very interesting to compare the maser positions with the map of the optical reflection nebula surrounding VY CMa (Herbig, 1972). The absolute 
position of the maser spots is not known to better than 5 " (Eliasson and Bartlett, 1969), but if we tentatively superpose the positions of expansion centre as given by the model of Moran et a1. (1977 b) and the star in Herbig's map as has been done in Figure 12 one sees that the maser spots fall in a very interesting part of the nebula where the optical polarization is particularly high.

It is interesting to note that the extent of the main-1ine oH sources is $\{0.2 "$ (Harvey et a1., 1974 b). It is very important to know the position of this emission relative to the positions of the $1612-\mathrm{MHz}$ emission and of the star. One would certainly expect it to be closer to the star than the $1612-\mathrm{MHz}$ emission.

\section{Carbon Stars}

The most "famous" object for microwave molecular lines in carbon stars is IRC +10216 (or CW Leo). It consists of a small ( $\sim 0.4^{\prime \prime}$ ), optically thick component at $375 \mathrm{~K}$ (Becklin et a1., 1969; Toombs et al., 1973). Spectra of the source suggest that it is a late-type carbon star, surrounding by obscuring dust presumably produced in the atmosphere of the star itself (Miller, 1970; Herbig and Zappala, 1970). The envelope gives rise to millimeter-wavelength emission from many molecules: CO (Solomon et al., 1971; Kuiper et al., 1976), $\mathrm{CN}$ and CS (Wilson et al., 1971; Turner et a1., 1973), HCN (Morris et a1., 1971; Wilson et al., 1973), $\mathrm{C}_{2} \mathrm{H}$ (Tucker et al., 1974), and SiS, SiO, and $\mathrm{HC}_{3} \mathrm{~N}$ (Morris et a1., 1975). The large velocity width $\left(24 \mathrm{~km} \mathrm{~s}^{-1}\right)$ seen in these lines strongly suggests expansion of the envelope. The size of the cloud emitting these molecular lines has been measured as $\left\{40^{\prime \prime}\right.$ for HCN and $\sim 2.1$ ' for CO (Wilson et al, 1973).

In Figure 13 the ${ }^{12} \mathrm{CO}$ and ${ }^{13} \mathrm{CO} \mathrm{J}=1-0$ lines as observed with the NRAO 11-m telescope towards the centre of $I R C+10216$ are shown (Kuiper et al., 1976). Kuiper et al. (1976) show that these observations are consistent with a partially resolved, expanding envelope, with a constant excitation temperature, constant radial expansion velocity, and an inverse-square-1aw density dependence (i. e. constant mass loss from the central star). The profile shapes are consistent with the ${ }^{12} \mathrm{Co}$ line being optically thick and the ${ }^{13} \mathrm{CO} 1$ ine thin. The expansion velocity of $\mathrm{CO}$ is $\sim 15 \mathrm{~km} \mathrm{~s}^{-1}$, whereas the other molecules have an expansion velocity of $\sim 12 \mathrm{~km} \mathrm{~s}^{-1}$ (Morris, 1975; Morris et a1., 1975). These results suggest that the expansion velocity increases slightly 
LSR VELOCITY, $\mathrm{km} / \mathrm{s}$

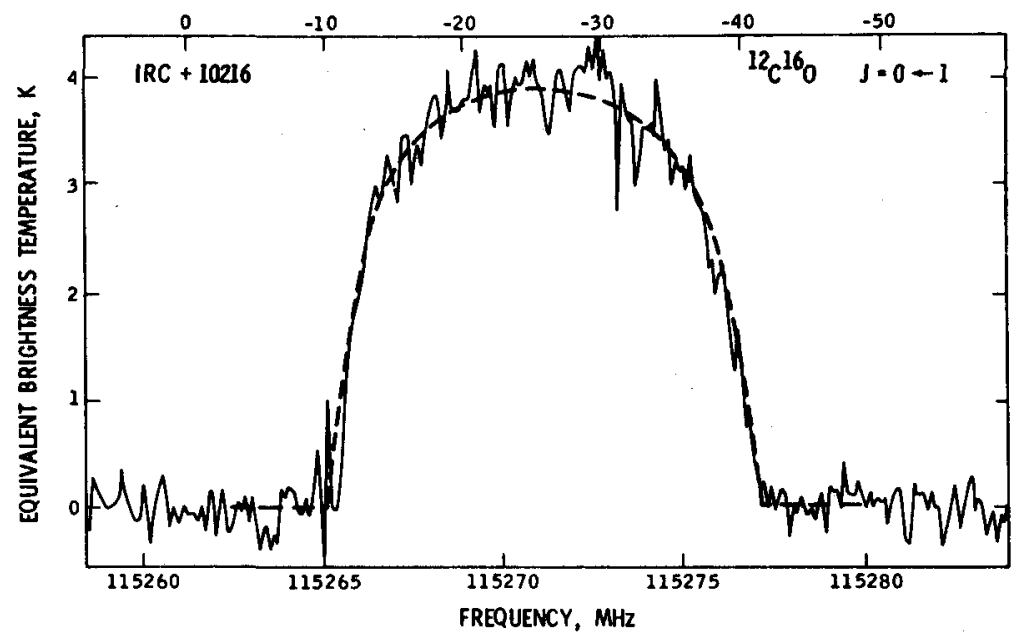

LSR VELOCITY, $\mathrm{km} / \mathrm{s}$

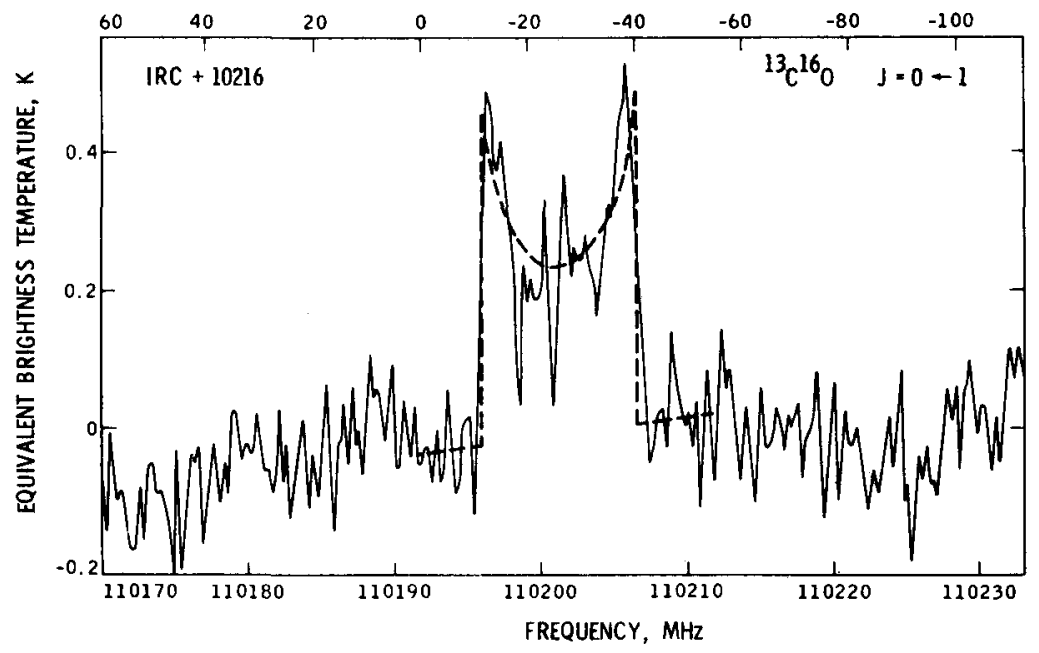

Fig. 13 Line profiles of $\mathrm{J}=1$ - 0 transition from ${ }^{12} \mathrm{CO}$ (top) and $13 \mathrm{CO}$ (bottom) at the central position of IRC+10216. The dashed lines represent a uniform mass outflow model in which the excitation temperature is assumed to be constant throughout the envelope. The 12 Co line is optica1ly thick and the ${ }^{3} \mathrm{CO}$ line optically thin. From Kuiper et al. (1976) 
with distance from the star. Kwan and Hill (1977) have proposed a model of the envelope around IRC+10216 based on observations of ${ }^{12} \mathrm{CO}$, ${ }^{13} \mathrm{CO}, \mathrm{H}^{12} \mathrm{CN}$, and $\mathrm{H}^{13} \mathrm{CN}$ lines. Assuming a distance of $200 \mathrm{pc}$ the deduced mass loss rate is $2 \times 10^{-5} \mathrm{M}_{\Theta} \mathrm{yr}^{-1}$ and the $[\mathrm{CO}] /\left[\mathrm{H}_{2}\right]$ abundance ratio about $8 \times 10^{-4}$, both uncertain to a factor of 2 .

Another object which is quite similar to IRC +10216 is CRL2688. Zuckerman et al. (1976) observed lines from $\mathrm{H}^{12} \mathrm{CN}, \mathrm{H}^{13} \mathrm{CN}, \mathrm{CS}$, and $\mathrm{HC}_{3} \mathrm{~N}$ from this object. These authors suggest that CRL 2688 is an evolved carbon star mainly on the basis of the optical and microwave spectra. They speculate that such objects may be the progenitors of planetary nebulae and they place CRL2688 quite late in this evolution.

\section{Acknowledgements}

I an grateful to D. Downes and G. Winnewisser who kindly supplied data in advance of publication. I also wish to thank W. A. Sherwood and $C$. M. Walmsley for critical reading of the manuscript.

\section{References}

Andersson, C., Johansson, L. E. B., Goss, W. M., Winnberg, A., and Nguyen-Q-Rieu: 1974 , Astron. Astrophys. 30, 475

Balister, M., Batchelor, R. A., Haynes, R. F., Knowles, S. H., McCulloch, M. G., Robinson, B. J., Wellington, K. J., and Yabsley, D. E.: 1977, Mon. Not. Roy. Astron. Soc. 180, 415

Baudry, A., LéSqueren, A. M., and Lepine, J. R. D.: 1977, Astron. As trophys. 54,593

Becklin, E. E., Frogel, J. A., Hyland, A. R., Kristian, J., and Neugebauer, G.: 1969, As trophys. J. 158, L133

Blair, G. N., and Dickinson, D. F.: 1977, Astrophys. J. 215, 552

Blerkom, D. Van, and Auer, L.: 1976, Astrophys. J. 204, 775

Bowers, P. F.: 1975, Astron. Astrophys. 39, 473

Bowers, P. F., and Kerr, F. J.: 1977, Astron. Astrophys. 57, 115

Buh1, D., Snyder, L. E., Lovas, F. J., and Johnson, D. R.: 1974, Astrophys. J. 192, 297

Buh1, D., Snyder, L. E., Lovas, F. J., and Johnson, D. R.: 1975, Astrophys. J. 201, L29

Caswe11, J. L.: 1974, in Galactic Radio Astronomy, eds. F. J. Kerr and S. C. Simonson III, p. 423

Davis, J. H., Blair, G. N., van Til1, H., and Thaddeus, P.: 1974, Astropnys. J. 190, L117

Dickinson, D. F., and Kleinmann, S. G.: 1977, Astrophys. J. 214, L135

Dickinson, D. F., Kollberg, E., and Yngvesson, S.: 1975, Astrophys. J. 199,131

Eliasson, B., and Bartlett, J. F.: 1969, Astrophys. J. 155, L79 
Elitzur, M., Goldreich, P., and Scoville, N.: 1976, Astrophys. J. 205,384

Geballe, T. R., and Townes, C. H.: 1974, Astrophys. J. 191, L37

Genzel, R., and Downes, D.: 1977 a, Astron. Astrophys., in press

Genze1, R., and Downes, D.: 1977 b, Astron. Astrophys. 61, 117

Goldreich, P., and Scoville, N.: 1976, Astrophys. J. 205, 144

Goon, G. , and Auman, J. R.: 1970, Astrophys. J. 161, 533

Hansen, S. S., Moran, J. M., Reid, M. J., Johnston, K. J., Spencer, J. H., and Walker, R. C.: 1977, Astrophys. J., in press

Harvey, P. M., Bechis, K. P., Wilson, W. J., and Bal1, J. A.: 1974 a, Astrophys. J. Suppl. 27, 331

Harvey, P. J., Booth, R. S., Davies, R. D., Whittet, D. C. B., and McLaughilin, W.: 1974 b, Mon. Not. Roy. Astron. Soc. 169, 545

Herbig, G. H.: 1969, Mém. Soc. Roy. Sci. Liège 19, 13

Herbig, G. H.: 1972 , Astrophys. J. 172,375

Herbig, G. H., and Zappala, R. R,: 1970, Astrophys. J. 162, L15

Hinkle, K. H., Barnes, T. G., Lambert, D. L., and Beer, R. : 1976, Astrophys. J. 210, L141

Hyland, A. R.: 1974, in Galactic Radio Astronomy, eds. F. J. Kerr and S. C. Simonson III, p. 439

Kaifu, N., Buh1, D., and Snyder, L. E.: 1975, Astrophys. J. 195, 359

Knowles, S. II. , and Batchelor, R. A.: 1976, Mon. Not. Roy. Astron. Soc. $174,69 \mathrm{P}$

Kuiper, T. B. H., Knapp, G. R., Knapp, S. L., and Brown, R. L.: 1976, Astrophys. J. 204,408

Kwan, J., and Hill, F.: 1977, Astrophys. J. 215, 781

Kwan, J., and Scovil1e, N.: 1974, Astrophys. J. 194, L97

Kwok, S.: 1976 , J. Roy. Astron. Soc. Can. 70, 49

Litvak, M. M.: 1969, Science 165,855

Litvak, M. M. , and Dickinson, D. F.: 1972, Astrophys. L. 12, 113

Masheder, M. R. W., Booth, R. S., and Davies, R. D.: 1974, Mon. Not. Roy. Astron. Soc. 166, 561

McGee, R. X., Balister, M., and Newton, L. M.: 1977 a, Mon. Not. Roy. Astron. Soc. 180,585

MeGee, R. X., Newton, L. M., and Brooks, J.W.: 1977 b, Mon. Not. Roy. Astron. Soc. 180, 91P

Miller, J. S.: 1970, Astrophys. J. 161, L95

Moran, J. M., Johnston, K. J., Spencer, J. H., and Schwartz, P. R.: 1977 a, Astrophys. J., in press

Moran, J. M., Ball, J. A., Yen, J. L., Schwartz, P. R., Johnston, K. J., and knowles, S. H: 1977 b, Ástrophys. J. 211, 160

Morris, M.: 1975, Astrophys. J. 197, 603

Morris, M., and Álcock, C.: 1977, Astrophys. J., in press

Morris, M., Gilmore, W. S., Palmer, P., Turner, B. E., and Zuckerman, B. M.: 1975, Astrophys. J. 199, L47

Morris, M., Zuckerman, B. M., Paimer, P., and Turner, B. E.: 1971, Astrophys. J . 170, L110

Olnon, F. M.: 1977, $\mathrm{PhD}$ Thesis, University of Leiden

Pataki, L., and Kolena, J.: 1974 , Bull. Am. Astron. Soc. 6, 340

Reid, M. J.: 1975, PhD Thesis, California Institute of Technology

Reid, M. J., and Dickinson, D. F.: 1976, Astrophys. J. 209, 505

Reid, M. J., Muhleman, D. O., Moran, J. M., Johnston, K. J., and Schwartz, P. R.: 1977 , Astrophys.'J. 214,60

Ridgeway, S. T., Wells, D. C., and Joyce, R. R.: 1977, Astron.J.82,414

Schwartz, P. R., Harvey, P. M., and Barrett, A. H.: 1974, Astrophys. J. 187,491

Slutz, S.: 1976, Astrophys. J. 210, 750

Snyder, L. E., and Buh1, D.: 1974, Astrophys. J. 189, L31

Snyder, L. E., and Buh1, D.: 1975, Astrophys. J. 197, 329

Solomon, P. M., Jefferts, K. B., Penzias, A. A., and Wilson, R. W.: 1971, Astrophys. J. 163, L53 
Toombs, R. I., Becklin, E. E., Frogel, J. A., Law, S. K., Porter, F. C., and Westphal, J. A.: 1973, Astrophys. J. 173, L71

Tucker, K. D., Kutner, M. L., and Thaddeus, P.: 1974, Astrophys. J. 193, L115

Turner, B. E., Zuckerman, B. M., Palmer, P., and Morris, M.: 1973, Astrophys. J. 186,123

Wallerstein, G.: 1975, Astrophys. J. Suppl. 29, 375

Wallerstein, G.: 1977, Astrophys. J. 211, 170

Wilson, W. J., and Barrett, A.H.: 1972, Astron. Astrophys. 17, 385

Wilson, W. J., Barrett, A. H., and Moran, J. M.: 1970, Astrophys. J. 160,545

Wilson, R. E., and Merrill, P. W.: 1942, Astrophys. J. 95, 248

Wilson, W. J., Schwartz, P. M., and Epstein, E. E.:1973, Astrophys. J. 183,871

Wilson, W. J., Schwartz, P. R., Neugebauer, G., Harvey, P. M., and Beckiin, E. E.: 1972, Astrophys. J. 177, 523

Wilson, R. W., Solomon, P. M., Penzias, A. A., and Jefferts, K. B.: 1971, Astrophys. J. 169, L35

Zuckerman, B., Gilra, D. P., Turner, B. E., Morris, M., and Palmer, P.:1976, Astrophys. J. 205, L15

Zuckerman, B., Palmer, P., Morris, M., Turner, B. E., Gilra, D. P., Bowers, P. F., and Gilmore, W.: 1977 , Astrophys. J.' 211, L97

\section{I S C US S I ON Of paper by WINNBERG:}

BAUD: How do you know the position of the OH maser spots with respect to the funny structure around VY CMa studied by Herbig?

WINNBERG: I simply superposed the VLBI map with the model fit by Moran et al. on Herbig's map bringing the centre of expansion to coincide with the star. However. I want to emphasize the uncertainty of the model fit. The actual position of the maser spots in the nebula has to await absolute OH VLBI position measurements.

HABING: Just a small addition. Baud and I have seen a few $1612 \mathrm{OH}$ masers with noticeable emission near the central velocity. 